\title{
The role of the molecular biology laboratory in the management of chronic hepatitis B and C
}

\author{
Peter Karayiannis \\ St George's University of London Medical School at the University of Nicosia, Cyprus
}

\begin{abstract}
Molecular biology techniques are routinely used nowadays to diagnose and evaluate antiviral treatment of patients with chronic hepatitis $\mathrm{B}(\mathrm{HBV})$ and hepatitis $\mathrm{C}$ virus (HCV) infections. Current tools at our disposal include tests that quantify the amount of circulating virus in the blood, techniques that can analyse genomic sequences to determine viral genotypes or subtypes, or determine amino-acid substitutions that may confer resistance to existing antiviral drugs. What is more, continuously evolving serological tests for the detection of viral antigens or their corresponding antibodies, have made diagnosis of disease as sensitive as possible. The present review will concentrate primarily on molecular diagnostics.
\end{abstract}

\section{Introduction}

Virological techniques, whether serological or molecularly based have been in existence now for more than 25 years, in relation to infection with either HBV or HCV. Both of these viruses cause chronic infection in $5 \%$ and in more than $70 \%$ of exposed adult individuals. Long-term carriage of both viruses and the ensuing immune-mediated damage to the liver can lead to the development of cirrhosis and hepatocellular carcinoma. As acute exposure to the viruses can go unnoticed in the absence of symptoms, chronic infection may take years to be diagnosed. Nevertheless, whether acute or chronic infection, the role of the laboratory is critical in the detection of viral antigens or antibodies, monitoring disease activity through the detection of markers of active viral replication such as HBV-DNA and HCV-RNA, and based on these findings,make a decision whether to initiate antiviral treatment or wait. If such treatment is initiated, then the effectiveness

Correspondence: Peter Karayiannis

E-mail: karayiannis.p@unic.ac.cy

(C) Copyright P. Karayiannis, 2013

Licensee PAGEPress, Italy

Thalassemia Reports 2013; 3(s1):e26

doi:10.4081/thal.2013.s1.e26

This article is distributed under the terms of the Creative Commons Attribution Noncommercial License (by-nc 3.0) which permits any noncommercial use, distribution, and reproduction in any medium, provided the original author(s) and source are credited.

Parts of this work were presented at the

" ${ }^{\text {rd }}$ Pan-European Conference on Haemoglobinopathies and Rare Anaemias", Limassol (Cyprus), 24-26 October 2012. of the drugs used is monitored by regular viral load measurements. Based on these measurements a response guided approach can be adopted whereby, if the treatment is ineffective then a decision to terminate treatment can be made (stopping rules) or continue with treatment but alter its duration.

Treatment duration in the case of $\mathrm{HBV}$ involves the use of nucleos(t)ide analogues, which in general require long-term treatment. In simplistic terms for the purposes of this review, HBeAg positive patients which do not undergo seroconversion to anti-HBe whilst on treatment, and anti-HBe patientswho need treatment, have to continue with the treatment for very long periods of time. How long this time can be is currently not defined, but as relapse (resumption of viral replication) is almost certain if treatment is discontinued, these patients are likely to be remain on treatment long-term until evidence to the contrary is forthcoming.

For chronic HCV patients treatment entails the use of a combination of drugs, namelypegylated interferon and the nucleoside analogue ribavirin. Up until recently this treatment resulted in a sustained virologic response (SVR) in about $50 \%$ of patients. However, SVR rates vary depending on the genotype of the virus. Analysis of the results indicated that patients with genotype (G) 1 and 4 infection had 40-45\% SVR rates, whilst those infected with $\mathrm{G} 2$ \& 3 had response rates exceeding $75 \%$. Moreover, it soon became apparent that the duration of treatment in the latter case could be curtailed to six months without compromising the results, unlike the case for G1 \& 4 patients who required the full twelve months of treatment.Thus, G1 \& 4 constitute the difficult to treat genotypes. More recently, two protease inhibitors have been licensed which target the non-structural protein 3 (NS3) which is responsible for the processing of the viral polyprotein after translation. The two drugs areBoceprevir and Telaprevir have been used in triple therapy regimens in phase III clinical trials and their results have been published recently. These trials have shown SVR rates ranging from $68 \%$ to $75 \%$ in $\mathrm{G} 1$ infected patients. As there are currently numerous other direct acting antivirals undergoing clinical testing, their evaluation becomes more and more dependent on laboratory findings relating to genotype assignment, viral load measurement and the possible development of resistance.

\section{Laboratory methods}

\section{Genotype assignment}

HBV has been divided into at least eight genotypes (A-H)based on phylogenetic analysis of sequences from different parts of the world, and some of these genotypes are further subdivided into a number of sub-genotypes. Similarly, HCV has a considerable amount of genetic variation which has allowed the division of virus isolates into six genotypes (G1-6) and over 100 subtypes. There are a number of commercially available kits for this purpose, particularly in relation to HCV infection, as genotype assignment is not a requirement for HBV treatment initiation. All of these target the 5 ' non-coding region (NCR) of 
the genome, and range from direct sequencing following polymerase chain reaction (PCR) amplification (Trugene), to reverse hybridization analysis using genotype-specific oligonucleotides (INNO-LiPa HCV II and Versant HCV version II), or genotype-specific real-time PCR (Abbott HCV II). These approaches are quite sensitive and misassignment of genotype is very low.

\section{Viral load measurements}

All methods available nowadays employ PCR approaches, whereby an amplicon is generated which is quantitatively measured in Real-Time. Measurements of viral load are essential in making a decision on whether to treat or not, in evaluating the effectiveness of treatment and in established breakthrough infections during treatment that may be attributable to development of resistance. Viral nucleic acids are normally isolated from serum and subjected to PCR, either directly as in the case of HBV or following reverse transcription to cDNA as in the case of HCV. Inclusion of known standards in each run allows the plotting of standard curves which are used by the instrument to calculate the amount of starting material.

There are a number of commercial kits available for real-time PCR and target-mediated amplification. They are fully or partly automated and avoid false positives due to the enclosed system approach that is employed during extraction and amplification which eliminates carryover contamination. The lower limit of detection (LLOD) with these assays ranges from $6-20 \mathrm{IU} / \mathrm{ml}$ in the case of HBV DNA detection, and $12-40 \mathrm{IU} / \mathrm{ml}$ in the case of HCV-RNA, with abroad dynamic range (up to $\mathrm{x} 108 \mathrm{IU} / \mathrm{ml})$. The LLOD should not be confused with the lower limit of quantitation (LLOQ) as these may not be the same for a given assay. The LLOQ is the lowest amount of nucleic acid that can be accurately quantitated as it falls on the linear part of the graph. The LLOD on the otherhand denotes the limit above which a sample is positive but cannot be quantitated and below which it is negative.

\section{Response definitions}

Failure to achieve a >2log decline in HCV RNA from baseline by week 12 is an indication for discontinuation of treatment, as the patient is unlikely to respond (null responders). On the other hand patients with undetectable HCV RNA by week 4 (RVR, rapid virological response) are more likely to achieve SVR. Patients who experience a $>2 \log$ decline in HCV RNA by week 12 are said to have an early virological response (EVR), but if they fail to clear HCV RNA by week 24 are referred to as partial responders. Those who are negative for HCV RNA at the end of treatment but become positive during the 24 week followup are known as relapsers.

In the case of HBV, successful antiviral treatment aims to achieve normalisation of ALT levels, suppression of HBV-DNA to undetectable levels (both in HBeAg-positive and -negative $\mathrm{CHB}$ ), loss of $\mathrm{HBeAg}$ with or without development of anti-HBe, and a decrease in the necroinflammatory score by $\geq 2$ points with no worsening in fibrosis. Loss of HBsAg with or without development of anti-HBs is a more desirable outcome but not so easily achievable.

\section{Sequencing studies}

\section{Quasispecies}

Manual sequencing techniques, eventually gave way to automatic sequencing approaches that allowed the generation and analysis of sequences for genotyping and clonal variation using phylogenetic programs. The RNA-dependent RNA polymerase (RdRp)of HCV lacks proofreading capacity resulting in the introduction of point mutations during RNA replication. As a result, the virus circulates in the blood of an infected individual as a swarm of closely related variants known as qua- sispecies. Similarly, although HBV is a DNA virus, its replication involves the use of an RNA intermediate (pre-genomic RNA) which is used as a template for the synthesis of the negative sense DNA stand of the genome by reverse transcription, a step which is prone to nucleotide misincorporations. Therefore, HBV has a higher mutation rate than other DNA viruses and has a quasispecies nature also. Some of the arising mutations are lethal and are lost, but others can be advantageous to both viruses. Such mutations may offer a replication advantage, permit escape from immune recognition and elimination, immune escape from vaccine induced antibodiesas in the case of $\mathrm{HBV}$, and finally lead to resistance to antiviral drugs. New generation sequencing technologies allow the accumulation of vast amounts of data, much more so than the information obtained by cloning/sequencing. Using such quasispecies analyses, it has been shown that at least $9 \%$ of treatment naïve patients can have pre-existing resistance mutations that would undermine antiviral therapy if instituted.

Sequencing studies have led to the identification of a number of important genomic mutations. For the purposes of this review, one example for each virus will be given relating to immune escape. The amino-terminal end of the envelope glycoprotein 2 of HCV contains a stretch of 27 aminoacids that show extreme hypervariability between isolates of the same genotype or subtype from different parts of the world. This is also true between quasispecies within an individual at a given time, but also at different times. This region is known as hypervariable region 1 (HVR 1), and it is now known that it comes under immune attack leading to selection of different quasispecies. This is thought to constitute a mechanism of viral persistence whereby the dominant quasispecies, once under attack by the humoral immune response, is cleared giving the chance to one of the minor ones to take over. This fight between the virus and the immune system is ongoing and explains the frequent fluctuations in liver transaminase levels associated with immune lysis of hepatocytes. Moreover, the variation seen in HVR 1 has made the formulation of a therapeutic vaccine against HCV very difficult indeed.

In the case of $\mathrm{HBV}$, in the anti-HBe positive phase following reactivation of the virus, the core protein which forms the nucleocapsid of the virus comes under immune attack. The majority of chronic HBV patients seen in clinics in Southern Europe and the Far East nowadays belong to this group which is often referred to as the HBeAg negative group. We know that these patients have active disease as a result of the presence of $\mathrm{HBV}$ which has mutations in the core promoter or the pre-core regions. In the absence of the tolerogenic effect of HBeAg, the core protein when proteolyticallyprocessed into peptides that are displayed on the hepatocyte surface in association with HLA class I antigens can come under immune attack. The variability seen in the core region of quasispecies from such a patient, is also an attempt by this virus to evade the immune response.

\section{Antiviral resistance}

The treatment of chronic HBV entails monotherapy with nucleos(t)ide analogues long term as already indicated. However, some of them appear to have a low genetic barrier which allows the emergence of resistance mutations that compromise any beneficial effects achieved up to that point. Breakthrough infection warrants switching to an alternative antiviral the efficacy of which is not affected by the resistance mutations. The role of the molecular biology laboratory in this respect proved invaluable following the introduction of drugs with a low barrier of resistance such as Lamivudine (LMV) and adefovir (ADV). Resistance mutations are not a problem with high genetic barrier drugs such as entecavir (ETV) and tenofovir (TNF). The rt/DNA polymerase domain contains at least 5 subdomains (A-E) which are spatially separated, but closely associated with the normal function of the protein. LMV resistance is associated with amino-acid substitutions primarily in subdomain $\mathrm{C}$ which contains the characteristic YMDD (tyrosine-methionine-aspartate-aspartate) motif of the catalytic site,and may be accompanied by others in subdomain B 
(Table1). The main substitutions include rtM204I (YIDD) and rt M204V (YVDD). The rtM204V substitution is a companied usually by the rtL180M mutationin subdomain B which is thought to restore replication competency. Emergence of resistance rises to $75 \%$ after 5 years of treatment. Prolonged treatment with adefovirleads to emergence of resistance with two main mutations; rtA181T/N and rtN236T which account for a $30 \%$ resistance rate after 5 years of use.One other substitution, rtA181T/N on its own, has been shown to confer cross-resistance to LMV and ADV.

ETV has an extremely low incidence of resistance in treatment naïve patients; no resistance was detected by 2 years of treatment and only $1.2 \%$ at 5 years. Resistance however does arise in patients with preexisting LMV resistant quasispecies, who initiall have effective suppression of virus replication. The emergence of resistance is more frequent in this setting rising from $6 \%$ at year 1 to $57 \%$ at year 6 . This requires the presence of rtM204V and rtL180M, but not rtM204I, and of an additional mutation at rtI169T, rtT184G, rtS202I or rt M250V for resistance against ETV to emerge.

Genotypic resistance against telbivudine (TBV) stands at $4.4 \%$ and $21.6 \%$ at year 1 and 2 respectively in $\mathrm{HBeAg}$ positive, whilst in $\mathrm{HBeAg}$ negative patients the figures are 2.7 and $8.6 \%$,rising further by the 3rd year and thereafter. The main mutation is the rtM204I alone or in combination with rtL180M, and therefore such isolates are cross-resistant to LMV also.

\section{No resistance to TFV has been reported so far even after 6 years of follow-up.}

Treatment of chronic HCV infection with the standard of care, that is pegylated interferon and ribavirin, does not lead to the emergence of easily identifiable resistance mutations, even in relapsing patients. However, in vitro experiments and clinical phase I studiesindicated the emergence of resistant viruses very quickly with direct acting antivirals such as telaprevir and boceprevir when used as monotherapy. These protease inhibitors select pre-existing resistant variants from the quasispecies pool within 1-2 weeks of starting treatment, and this is the case with newer antivirals which are currently at different stages of clinical evaluation. The use of RBV in combination regimens appears to be important in minimising resistance at $2 \%$ in treble therapy regimens (with pegylated interferon) as opposed to 26\% in RBV free use.After stopping therapy, resistant viruses are overtaken once again by the wild type. Amino-acid substitutions that confer resistance and cross resistance between TPV and B0C, include R155K, T54S/A, V36M and A156T (Table 2).

\section{Conclusions}

In summary, the role of the molecular biology laboratory in the management of chronic HBV and HCV infections has become very importants, particularly when it comes down to monitoring of antiviral treatment. As the importance of genomics, proteomics, metabonomicsetc becomes clear, so will the dependence on the laboratory become increasingly all the more necessary.

Table 1. Amino-acid substitutions in the polymerase/rtregion of $\mathrm{HBV}$ that confer resistance or cross-resistance to nucleos(t)ide analogues. $\mathrm{ND}=$ none demonstrated.

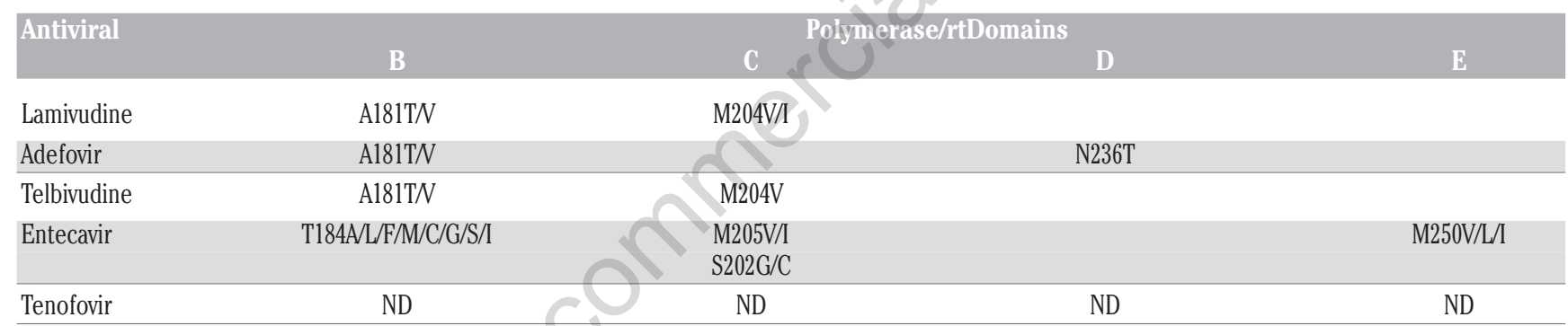

Table 2. Amino-acid substitutions in the NS3 serine protease of HCV which confer resistance to linear and macrocyclic inhibitors. NS4 is a co-factor required for efficient proteolytic processing of the viral polyprotein.

\begin{tabular}{|c|c|c|c|c|c|}
\hline \multirow[t]{2}{*}{ Targeted protein } & \multirow{2}{*}{ Mutation } & \multirow[b]{2}{*}{ Telaprevir } & \multicolumn{2}{|c|}{ NS3/NS4 Proteas } & \multirow[b]{2}{*}{$\begin{array}{l}\text { Macrocyclic } \\
\text { TMC-435 }\end{array}$} \\
\hline & & & $\begin{array}{l}\text { Linear } \\
\text { Boceprevir }\end{array}$ & BI-201335 & \\
\hline \multirow[t]{8}{*}{ NS3 } & $\mathrm{R} 155 \mathrm{~K}$ & $\sqrt{ }$ & $\sqrt{ }$ & $\sqrt{ }$ & \\
\hline & T54S/A & $\sqrt{ }$ & $\sqrt{ }$ & & \\
\hline & V36M & $\sqrt{ }$ & $\sqrt{ }$ & & \\
\hline & $\mathrm{A} 156 \mathrm{~T}$ & $\sqrt{ }$ & $\sqrt{ }$ & & \\
\hline & V170A & $\sqrt{ }$ & $\sqrt{ }$ & & \\
\hline & V55A & & $\sqrt{ }$ & & \\
\hline & D168V/A & & & $\sqrt{ }$ & $\sqrt{ }$ \\
\hline & F43S & & & & $\sqrt{ }(\mathrm{G} 2)$ \\
\hline
\end{tabular}

\section{Oral presentation}

Oral presentation is available online 